\title{
NF-KB-mediated IncRNA AC007271.3 promotes carcinogenesis of oral squamous cell carcinoma by regulating miR-125b-2-3p/Slug
}

\author{
Ze-nan Zheng ${ }^{1}$, Guang-zhao Huang ${ }^{1}$, Qing-qing Wu' ${ }^{1}$, Heng-yu Ye ${ }^{1}$, Wei-sen Zeng ${ }^{2}$ and Xiao-zhi Lv ${ }^{1}$
}

\begin{abstract}
Oral squamous cell carcinoma (OSCC) is the most common oral cancer. The molecular mechanisms of this disease are not fully understood. Our previous studies confirmed that dysregulated function of long non-coding RNA (IncRNA) AC007271.3 was associated with a poor prognosis and overexpression of AC007271.3 promoted cell proliferation, migration, invasion, and inhibited cell apoptosis in vitro, and promoted tumor growth in vivo. However, the underlying mechanisms of AC007271.3 dysregulation remained obscure. In this study, our investigation showed that AC007271.3 functioned as competing endogenous RNA by binding to miR-125b-2-3p and by destabilizing primary miR-125b-2, resulted in the upregulating expression of Slug, which is a direct target of miR-125b-2-3p. Slug also inhibited the expression of E-cadherin but $\mathrm{N}$-cadherin, vimentin, and $\beta$-catenin had no obvious change. The expression of AC007271.3 was promoted by the canonical nuclear factor-kB (NF-KB) pathway. Taken together, these results suggested that the classical NF-KB pathway-activated AC007271.3 regulates EMT by miR-125b-2-3p/Slug/E-cadherin axis to promote the development of OSCC, implicating it as a novel potential target for therapeutic intervention in this disease.
\end{abstract}

\section{Introduction}

Oral squamous cell carcinoma (OSCC) is the most common type of oral cancer ${ }^{1}$. Tobacco, alcohol, betel quid, and human papillomavirus infection are the major risk factors for $\mathrm{OSCC}^{2}$. Despite recent remarkable advances in the treatment of OSCC in surgery, chemotherapy, radiotherapy and targeting therapy, the 5years survival rate is still $<60 \%$ owing to tumor recurrence and metastasis ${ }^{3,4}$. Therefore, understanding the detailed mechanisms of OSCC tumorigenesis and development will facilitate the establishment of new effective therapeutic alternatives in order to improve the curative effect and life quality.

\footnotetext{
Correspondence: Wei-sen Zeng (zengws@smu.edu.cn) or

Xiao-zhi Lv (Ixzsurgeon@126.com)

${ }^{1}$ Department of Oral \& Maxillofacial Surgery, NanFang Hospital, Southern Medical University, Guangzhou, People's Republic of China

2Department of Cell Biology, School of Basic Medical Science,

Southern Medical University, Guangzhou, People's Republic of China

Edited by B. Rotblat
}

LncRNAs are a group of genes longer than 200 nucleotides, which were considered as "functionless genes" in the past ${ }^{5}$. Over the past decade, numerous studies have shown that lncRNAs actually play important roles in various diseases, including cancers ${ }^{6,7}$. LncRNAs can function as tumor suppressors or oncogenes, transcriptional regulation, histone modification elements, splicing, and so on, which would promote or inhibit the OSCC progression ${ }^{8-10}$.

AC007271.3 is a 382-nucleotides-long lncRNA located on chromosome 2. Our previous study identified that AC007271.3 was significantly upregulated in OSCC than in adjacent normal tissues (ANTs) by microarray. Higher expression of AC007271.3 in serum was related with clinical stage and poor prognosis ${ }^{11}$. Further study showed that AC007271.3 mainly enriched in the cytoplasm and promoted OSCC cells proliferation, migration, invasion, and inhibited cell apoptosis in vitro, and promoted tumor growth in vivo ${ }^{12}$. However, the underlying mechanisms of

\section{(c) The Author(s) 2020}

(c) Open Access This article is licensed under a Creative Commons Attribution 4.0 International License, which permits use, sharing, adaptation, distribution and reproduction cc) in any medium or format, as long as you give appropriate credit to the original author(s) and the source, provide a link to the Creative Commons license, and indicate if changes were made. The images or other third party material in this article are included in the article's Creative Commons license, unless indicated otherwise in a credit line to the material. If material is not included in the article's Creative Commons license and your intended use is not permitted by statutory regulation or exceeds the permitted use, you will need to obtain permission directly from the copyright holder. To view a copy of this license, visit http://creativecommons.org/licenses/by/4.0/. 
AC007271.3, promoting OSCC carcinogenesis remain to be excavated.

MicroRNAs (miRNAs) are a group of non-coding RNAs with a length of $\sim 22$ nucleotides, which can direct the RNA-induced silencing complex to suppress the mRNA translation or degrade them by targeting the $3^{\prime}$-untranslated region ( $3^{\prime}$-UTR) of $\mathrm{mRNA}^{13}$. Accumulating evidence proved that miRNAs were related to cancers, and several miRNAs have been researched as therapeutic molecular ${ }^{14}$. Recent studies indicated that lncRNAs located in the cytoplasm may play as competing endogenous RNA (ceRNA) of miRNAs ${ }^{15}$. This phenomenon was widely discovered $^{16,17}$ and also reported in OSCC ${ }^{18,19}$.

According to the location of AC007271.3, we speculated that it could play as a ceRNA of miRNAs. Further investigation verified that AC007271.3 increased Slug expression by regulating miR-125b-2-3p and promoted the migration and invasion in OSCC cells. What's more, classical nuclear factor- $\mathrm{kB}$ (NF- $\mathrm{kB}$ ) pathway was found to activate the expression of AC007271.3. Our findings provided a novel insight into the mechanisms of AC007271.3 in OSCC development, which suggested AC007271.3 could be a possible target for OSCC therapy.

\section{Materials and methods}

\section{Ethics statement and tissue samples}

The OSCC tumor tissues and matched ANTs in this study were collected from 82 patients with OSCC under surgery at the Department of Oral and Maxillofacial Surgery, Nanfang Hospital, Guangzhou, Guangdong Province after obtaining informed consent. The diagnosis was pathologically confirmed by the Department of Pathology, Nanfang Hospital. ANTs located at least $1.5 \mathrm{~cm}$ from the edge of the tumor were defined as normal control. This study was approved by Research Scientific Ethics Committee of NanFang Hospital (NFEC-2018027).

\section{Cell culture}

The human tongue squamous cell lines (SCC9 and SCC15), normal human oral keratinocyte cell line (HOK), and human embryonic kidney cell line (HEK 293 T) were obtained from the Institute of Antibody Engineering, Southern Medical University (Guangzhou, China). All cells were cultured in Dulbecco's Modified Eagle's Medium (DMEM) (Gibco, NY, USA) with 10\% Fetal bovine serum (ExCell Bio, Shanghai, China) and $100 \mathrm{U} / \mathrm{ml}$ Penicillin-Streptomycin (Invitrogen, CA, USA) at $37^{\circ} \mathrm{C}$ in a humidified $5 \% \mathrm{CO}_{2}$.

\section{Expression plasmid, RNA oligonucleotides, and transfection}

The AC007271.3 overexpression vector pcDNA3.1 (+)-AC007271.3 and its corresponding control vector
pcDNA3.1(+), specific siRNAs targeting AC007271.3 (siAC007271.3), Slug (si-Slug 1, si-Slug 2, si-Slug 3) and the scramble negative control siRNA (si-AC007271.3 NC, siSlug NC) were obtained from Sangon Biotech Corp., Ltd. (Shanghai, China). The miR-125b-2-3p inhibitor, miR125b-2-3p mimic, and the negative control (miR-125b-23p mimic NC, miR-125b-2-3p inhibitor NC) were purchased from RiboBio Corp, Ltd. (Guangzhou, China). Transfections were performed with Lipofectamine 3000 reagent (Invitrogen, CA, USA) following the manufacturer's protocols.

\section{RNA extraction and qRT-PCR}

Total cellular RNA from the cells was extracted by RNA isolater Total RNA Extraction Reagent (Vazyme, Nanjing, China) following the manufacturer's instructions. HiScript QRT SuperMix for qPCR (+gDNA wiper) (Vazyme, Nanjing, China) and miRNA 1st Strand cDNA Synthesis Kit (by stem-loop) (Vazyme, Nanjing, China) were used to generate cDNA. With ChamQ® ${ }^{\circledR}$ SYBR ${ }^{\circledR}$ qPCR Master Mix (Vazyme, Nanjing, China), quantitative real-time polymerase chain reaction (qRT-PCR) was performed on the Real-Time PCR detection system (Applied Biosystems, CA, USA). All expression levels were normalized against the GAPDH or U6 (for miRNA) mRNA level. The primer sequences are listed in Supplementary Table S1.

\section{mRNA stability assay}

The pcDNA3.1(+)-AC007271.3 plasmids were transfected into OSCC cells as described above. After 48 hours, actinomycin D (Sigma-Aldrich, St. Louis, MO, USA) was added to the culture medium at a concentration of $5 \mu \mathrm{g} /$ $\mathrm{mL}$, followed by incubation for 0 hour, 2 hours, 4 hours, 6 hours, or 8 hours. Pri-miR-125b-2 mRNA stability in the actinomycin $\mathrm{D}$ treatment group was analyzed by qRTPCR.

\section{Dual-luciferase reporter assay}

PmiRGLO dual-luciferase miRNA Target Expression Vector (Promega, WI, USA) was used to construct dualluciferase reporter and quantitatively evaluate the translational regulation activity of miR-125b-2-3p. The features list and map of this empty vector were showed in Supplementary Fig. S1. The predicted binding sites between miR-125b-2-3p and AC007271.3 (or the 3'-UTR of Slug mRNA) were inserted into the multiple cloning site region to construct the wild-type vectors (AC007271.3-WT, Slug-WT). Then the mutant type vectors (AC007271.3MUT, Slug-MUT) were obtained from the wild-type vectors by replacing the targeted sites (Replaced ACTTGTG with TCATCTC for AC007271.3-MUT, replaced ACTTGTGA with ACTGTCGA for Slug-MUT). All vectors were constructed by TsingKe Biological 
Technology Corp, Ltd. (Beijing, China). After cotransfecting with miR-125b-2-3p mimic into HEK $293 \mathrm{~T}$ cells for 48 hours, the Luc-Pair Dual-Luciferase Assay Kit 2.0 (GeneCopoeia, MD, USA) was used to detect the firefly and renilla luciferase activity. Renilla luciferase activity was used as control. For the core promoter identification, pGL3 vectors which contain the promoter fragments and phRL-TK vector were cotransfected into HEK $293 \mathrm{~T}$ cells for 48 hours and the following steps were the same.

\section{RNA pull-down assay}

Purified RNAs were biotin-labeled using Pierce RNA 3'End Desthiobiotinylation Kit (Thermo Fisher Scientific, CA, USA). Biotin-labeled wild-type miR-125b-2-3p (miR-125b-23p-Bio), Biotin-labeled mutant type miR-125b-2-3p (miR125b-2-3p-Bio-MUT) and negative control (NC-Bio) were incubated with SCC9 cell lysates overnight at $4{ }^{\circ} \mathrm{C}$. Magnetic beads were added to each mixture for 1 hour at room temperature. The eluted RNAs were detected by qRT-PCR.

\section{Western blot and antibody}

Protein obtained from cells or tissues were separated on $10 \%$ sodium dodecyl sulphate-polyacrylamide gel $(16 \mathrm{~mA}$, $1.5 \mathrm{~h}$ ) and then transferred to polyvinylidene fluoride membrane (100 V, 1.5 h) (Bio-Rad Laboratories). After blocking (5\% non-fat dry milk in phosphate-buffered saline (PBS) Tween) for 2.5 hours at room temperature, the primary antibodies (Supplementary Table S2) were used for incubation at $4{ }^{\circ} \mathrm{C}$ overnight and the secondary antibodies were incubated one hour at room temperature. ECL reagent (Millipore, Bedford, MA) was used for chemiluminescence. Molecular weight markers (Thermo Fisher Scientific, CA, USA, \#26616) was used as size standards in this assay.

\section{Cell migration and matrigel invasion assay}

OSCC cells transfected with RNA oligonucleotides or plasmid for $48 \mathrm{~h}$ were digested and resuspended in DMEM culture. In all, $5 \times 10^{4}$ cells were seeded into the upper chambers of 8- $\mu \mathrm{m}$ transwell inserts (Corning, NY, USA), and $500 \mu \mathrm{l}$ DMEM containing 20\% FBS was added to the lower chamber. After 36 hours' incubation, the membranes were fixed with methanol and stained with $0.1 \%$ crystal violet. Removed the cells on the top of the membranes and then counted the number of cells attached to the membranes under an inverted microscope. For invasion assay, matrigel (Corning, NY, USA) was diluted with DMEM (1:10) and covered the top membranes before cells seeding. Other steps were the same as migration assay.

\section{H\&E and IHC staining}

For histological examination, tissues were fixed with $4 \%$ paraformaldehyde at room temperature for 24 hours and washed with $70 \%$ alcohol. All tissues were embedded in paraffin and cut into sections ( $4 \mu \mathrm{m}$ thick). Hematoxylin and eosin were used for Hematoxylin and Eosin (H\&E) staining. For immunohistochemical (IHC) staining, after dewaxing, washing, rehydration, antigen retrieval, and endogenous peroxidase blocking following the manufacturer's protocols. Antibody was used to incubate at $4{ }^{\circ} \mathrm{C}$ overnight. Secondary biotinylated conjugated goat anti-rabbit antibody was incubated at room temperature for 30 mins. DAB (3,3'-diaminobenzidine) were used as chromogens and hematoxylin used as counterstains. Images were acquired under the light microscope (Leica, DM 2500, Wetzlar, Germany). The comparison of staining results between tumor tissues and ATNs was performed by two independent pathologists who were blinded to the clinical information of patients. The scoring criteria of staining were as follow, first was staining intensity: 0-none staining, 1-light yellow, 2-deep yellow, or 3brown; and the second criterion was staining cells proportion: $0(<5 \%), 1(5-25 \%), 2(25-50 \%), 3(51-75 \%)$, or 4 $(>75 \%)$. The product of the two scores was considered as the final score. The final scores were divided into two levels: 0-5 (Slug low expression) and $>5$ (Slug high expression).

\section{Animal experiment}

Lentivirus-NC and lentivirus-AC007271.3 were purchased from Obio Technology Corp., Ltd. (Shanghai, China) and transfected into SCC9 according to the operating instructions. Puromycin was used to select the AC007271.3 stably expressed cells (SCC9-AC007271.3) and its control cells (SCC9-NC). Five-weeks-old female BALB/c nude mice were randomly divided into three groups (Blank, SCC9-NC and SCC9-AC007271.3). Approximately $1 \times 10^{6} \quad$ SCC9AC007271.3 cells or SCC9-NC cells were resuspended in $500 \mu \mathrm{L}$ of PBS and injected via tail vein. Injection of $500 \mu \mathrm{L}$ of PBS without cells suspension was regarded as blank group. After 8 weeks, all mice were killed, and the visible lung metastases were counted. Lung metastases were excised for HE or IHC staining. The expression of AC007271.3 and miR-125b-2-3p were detected by qRT-PCR and the expression of Slug and E-cadherin were inspected by western blot. This animal experiment was approved by Southern Medical University Experimental Ethics Committee (L2018199), and all BALB/c nude mice were purchased from the Animal Care Unit of Southern Medical University.

\section{AC007271.3-promoter region cloning}

The sequences of the 2000bp before the $5^{\prime}$-UTR of AC007271.3 were regarded as promoter region and obtained from Ensemble database (http://asia.ensembl.org/index. $\mathrm{html})$. According to the sequences, we designed several primers with specific restriction enzyme protection sites of KpnI and Bgl II, respectively, for amplification the fragments $(-1998 /-2,-1508 /-2,-1000 /-2,-519 /-2)$. Genomic DNA was used as a template to amplify by KOD-Plus-Neo (TOYOBO, Osaka, Japan) amplification enzyme. The 
reaction conditions were set following the instruction. $1 \%$ agarose gel electrophoresis was performed to select the bands in correct size and confirmed through sequencing (Sangon, Shanghai, China). The pGL3-Basic vector and correct fragments were digested with KpnI (TakaraBio, Dalian, China) and Bgl II (TakaraBio, Dalian, China) restriction enzymes. DNA ligation kit ver.2.1 (TakaraBio, Dalian, China) was used to connect the fragments and pGL3-Basic vector. DH5 $\alpha$ competent cells were used for transformation. After the monoclonal colony sequence confirmation, the plasmids were extracted by using HiPure Plasmid EF Mini Kit (Magen, Guangzhou, China) for identification of core promoter of AC007271.3 by Dual-luciferase reporter assay.

\section{Chromatin immunoprecipitation (ChIP) assay}

ChIP assay were carried out by Magna ChIP ${ }^{\mathrm{TM}}$ A / G Kits (Merck, Darmstadt, German) according to the manufacturer's protocols. In brief, SCC9 cells were crosslinked with $1 \%$ formaldehyde, glycine quenching, and lysed. The nuclear DNA was fragmented by sonication. NFKB1 antibody was used for immunoprecipitation of crosslinked protein/DNA. Protein/DNA complexes were eluted and reverse crosslinked into free DNA. Purified DNA was used for qPCR analysis to detect the enrich fragments.

\section{Bioinformatics analysis}

Coding Potential Caculator (http://cpc.cbi.pku.edu.cn/) were performed to predict the protein-coding ability of AC007271.3. LncBase Predicted v.2 database (http:// carolina.imis.athena-innovation.gr/diana_tools/web/index. $\mathrm{php} ? \mathrm{r}=$ lncbasev2\%2Findex-predicted) were used to predict the potential miRNAs regulated by AC007271.3. TargetScan (http://www.targetscan.org/) were carried out to predict the target genes of miRNAs. The transcriptome profiling datum of OSCC were downloaded from The Cancer Genome Atlas (TCGA) database (https://portal.gdc.cancer.gov/) through GDC Data Transfer Tool. The Slug mRNA sequence data and relevant clinical information of 319 cases of OSCC and 32 cases of cancer ANTs were extracted from transcriptome profiling by $\mathrm{R}$ software (Version 3.6.1). The miR-125b-2-3p expression analysis results of OSCC and the GEO accession number (GSE45238) were obtained from dbDEMC 2.0 (https://www.picb.ac.cn/dbDEMC/). The miRNA sequence data of GSE45238 were downloaded from Gene Expression Omnibus (GEO) database (https://www.ncbi.nlm.nih.gov/ geo/query/acc.cgi?acc $=$ GSE45238) and extracted the expression data of miR-125b-2-3p. Gene-regulation online website (http://gene-regulation.com/pub/programs.html) and JASPAR database (http://jaspar.genereg.net/) were used for transcription factors prediction.

\section{Statistical analysis}

All experimental assays were performed in triplicate. All data were presented as mean \pm standard deviation $(\mathrm{SD})$ of triplicate replicates and analyzed by SPSS statistics 20.0 (Chicago, USA). All statistical charts were manufactured in Graphpad Prism 7.0 (CA, USA). Student's $t$ test was performed to compare the differences between the two groups. The correlation between miR-125b-2-3p (or Slug) and clinicopathological features was analyzed by using Fisher's exact tests. Pearson's correlation coefficient analysis was used to analyze the correlation between AC007271.3 and miR-125b2-3p. Kaplan-Meier curve was applied for detecting the comparison of overall survival in two groups.

\section{Results}

\section{Negative relation between AC007271.3 and miR-125b-2-3p expression}

Our previous study showed that AC007271.3 could promote the proliferation, migration, and invasion in $\mathrm{OSCC}^{12}$. We first predict the protein-coding potential of AC007271.3 by using Coding Potential Calculator. As exhibited in Supplementary Fig. S2, AC007271.3 had no apparent proteincoding ability. In recent years, cumulative evidence revealed that lncRNAs are vital in physiopathological processes partially by interacting with miRNAs. According to online platform LncBase Predicted v.2 database, three miRNAs (miR-4801, miR-1301-3p, miR-125b-2-3p), which contained the complementary sequences with AC007271.3 were predicted. To verify which miRNA or miRNAs were the potential target of AC007271.3, we overexpressed (Fig. 1a) or knocked down (Fig. 1b) AC007271.3 in two OSCC cell lines, respectively. The expression levels of three miRNAs were detected by qRT-PCR and the results showed that AC007271.3 could negatively regulate miR-125b-2-3p expression but induced inconsistent expression changes of miR-4801 and miR-1301-3p (Fig. 1c-d). On the other hand, overexpression of miR-125b-2-3p (Fig. 1e) resulted in a decrease of AC007271.3 (Fig. 1f), which indicated that AC007271.3 and miR-125b-2-3p could influence the expression with each other.

\section{AC007271.3 destabilized pri-miR-125b-2 and sponged with miR-125b-2-3p}

Mature miR-125b-2-3p was produced by primary miRNA125b-2 (pri-miR-125b-2), which also contains the predicted complementary sequences with AC007271.3. In order to explore whether AC007271.3 could downregulate miR-125b-2-3p by decreasing pri-miR-125b-2 or not, AC007271.3 was overexpressed or knocked down in OSCC cells and the results showed that AC007271.3 was also negatively correlated with pri-miR-125b-2 (Fig. 2a, b). The mRNA stabilization assays further confirmed that overexpression of AC007271.3 enhanced the degradation of primiR-125b-2 (Fig. 2c, d).

Furthermore, dual-luciferase reporter assays show that a significant decrease of luciferase activity was observed in the AC007271.3 wild-type's group but not in the mutant type's 

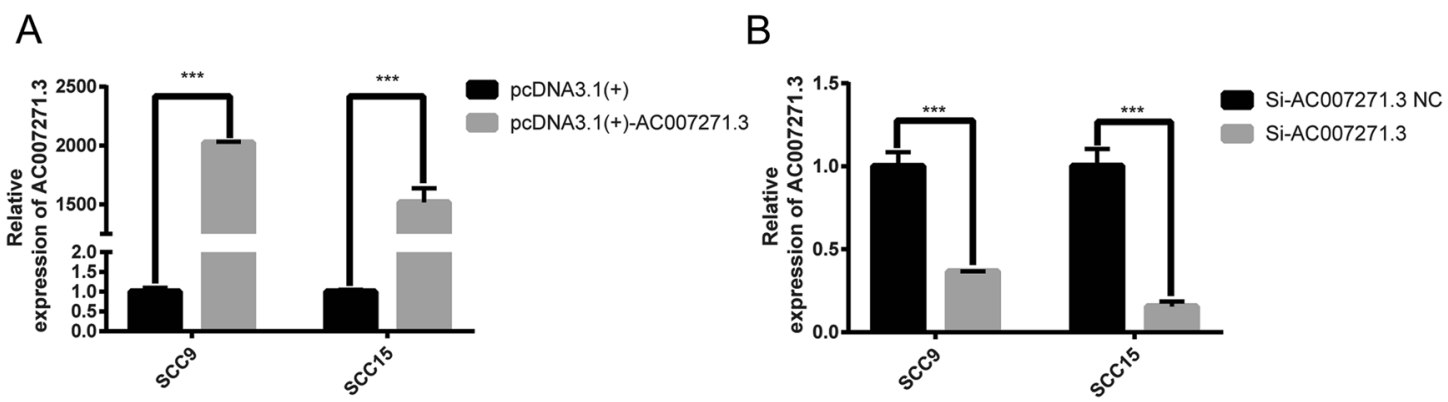

C
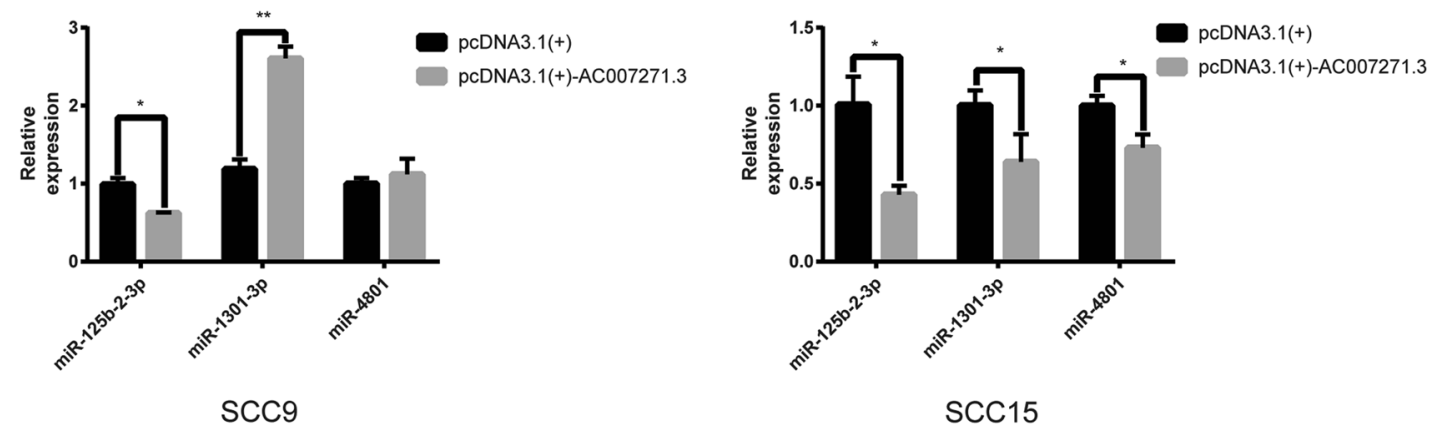

D
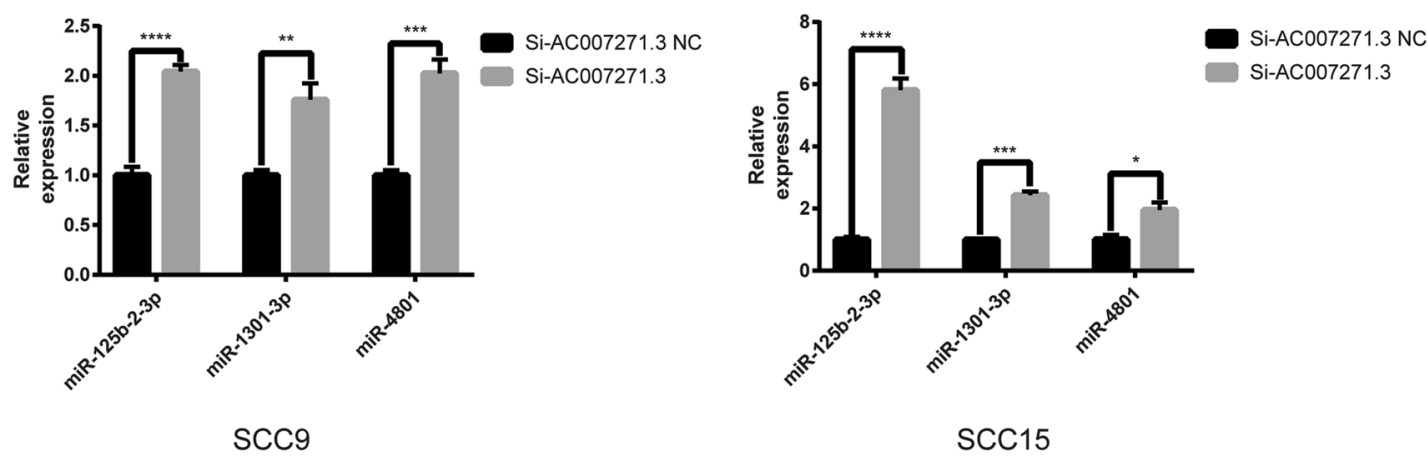

$\mathrm{E}$

$\mathrm{F}$
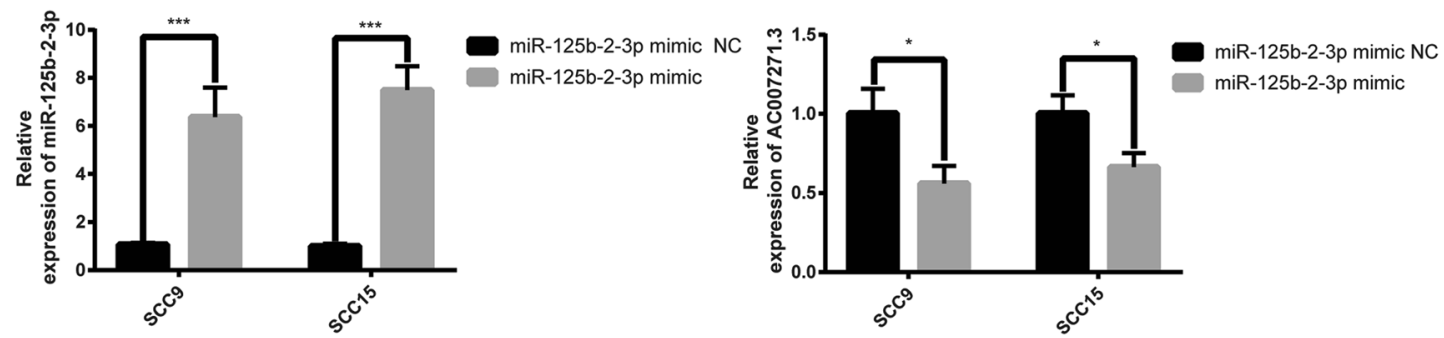

Fig. 1 AC007271.3 and miR-125b-2-3p regulated with each other. a The overexpression efficiency of pCDNA3.1(+)-AC007271.3 in SCC9 and SCC15 cells. b The knockdown efficiency of siRNA-AC007271.3 in SCC9 and SCC15 cells. c The expression of three predicted miRNAs after overexpressing AC007271.3. MiR-125b-2-3p were both significantly downregulated in SCC9 and SCC15. $\mathbf{d}$ The expression of three predicted miRNAs after knocking down AC007271.3 expression. MiR-125b-2-3p were the most significantly upregulated miRNA both in SCC9 and SCC15. e The overexpression efficiency of miR-125b-2-3p mimic in SCC9 and SCC15 cells. $\mathbf{f}$ The upregulation of miR-125b-2-3p inhibited the expression of AC007271.3. ${ }^{*} p<0.05,{ }^{* *} p<0.01,{ }^{* * *} p<0.001,{ }^{* * *} p<0.0001$. 

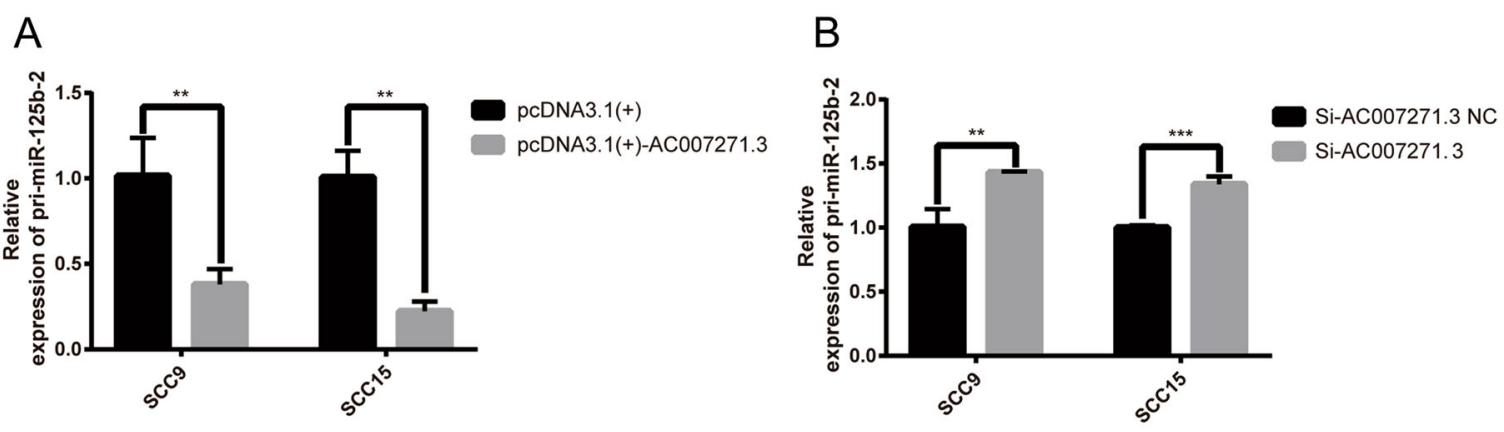

C

F
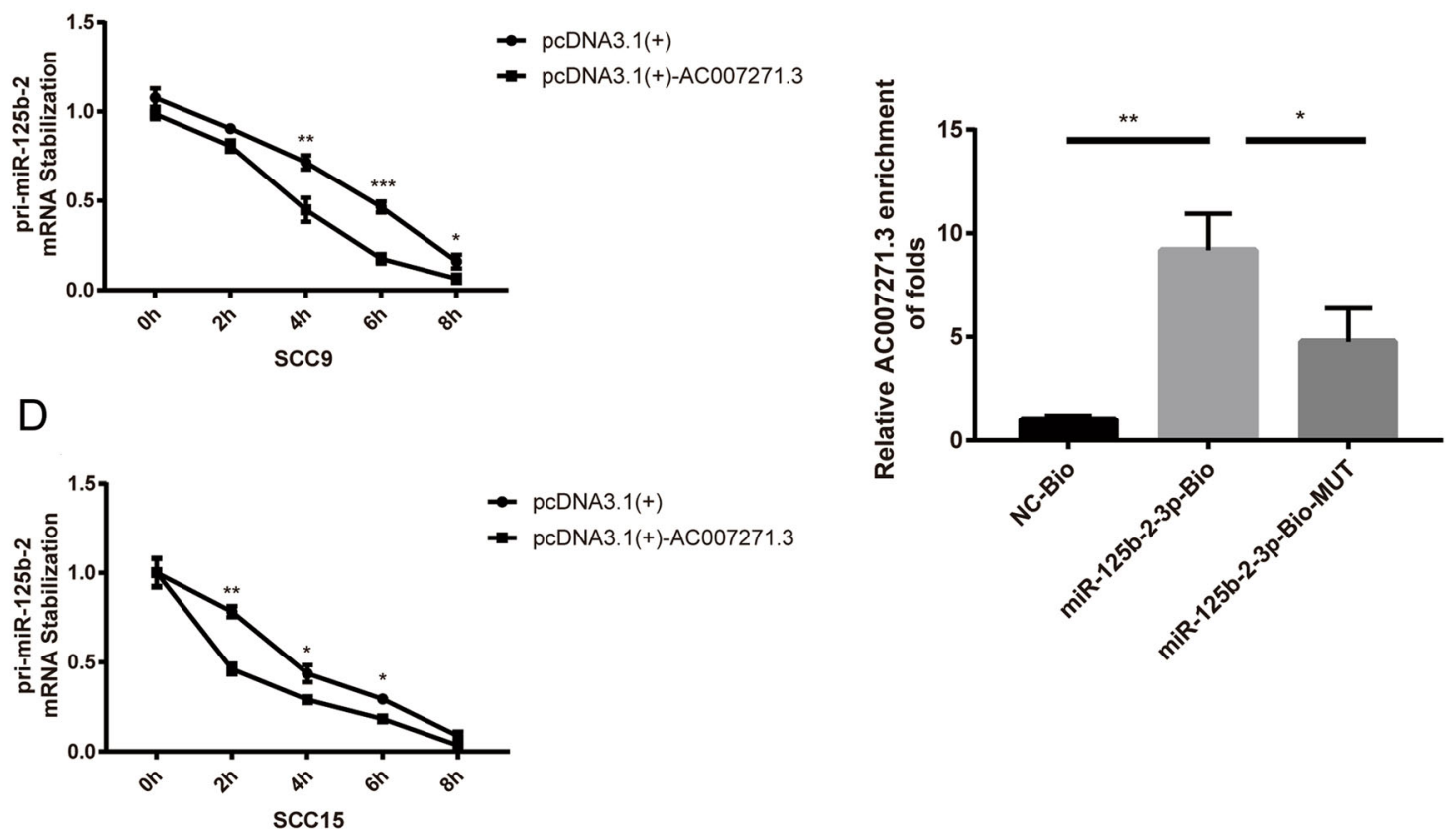

pcDNA3.1(+)

- pcDNA3.1(+)-AC007271.3

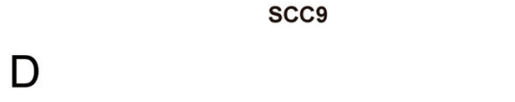


group (Fig. 2e) and the RNA pulldown assay exhibited that higher enrichment of AC007271.3 was observed in biotinylated miR-125b-2-3p probe than in control probe or biotinylated miR-125b-2-3p mutant probe (Fig. 2f). These results further confirmed that AC007271.3 binds directly with miR125b-2-3p.

\section{Overexpression of miR-125b-2-3p inhibited migration and invasion in OSCC}

To investigate the roles of miR-125b-2-3p in OSCC carcinogenesis, first, the relative expression levels of miR$125 \mathrm{~b}-2-3 \mathrm{p}$ in OSCC cells were detected by qRT-PCR. The results showed that the expression levels of miR-125b-2$3 p$ were significantly lower in OSCC cells than in HOK cells (Fig. 3a). What's more, compared with the matched ANTs, the expression levels of miR-125b-2-3p were downregulated in 82 OSCC tissues (Fig. 3b). A similar analysis result was also acquired from GEO database (Fig. $3 \mathrm{c})$. We further investigated the association between miR125b-2-3p expression and clinicopathological features of 82 OSCC patients. The results indicated that the expression of miR-125b-2-3p was negatively correlated with TNM classification $(p=0.0429)$ and lymph node metastasis $(p=0.0259)$ in OSCC patients (Supplementary Table S3).

Considered that miR-125b-2-3p was downregulated in OSCC tissues and cells, we next investigated the effects of miR-125b-2-3p overexpression on OSCC cell phenotypes. The results showed that miR-125b-2-3p mimic inhibited the ability of migration and invasion in SCC9 and SCC15 (Fig. 3D), whereas miR-125b-2-3p inhibitor (Fig. 3e) displayed the opposite results (Fig. 3f).

\section{Slug acted as a target gene of miR-125b-2-3p}

Plenty of evidence showed that miRNAs could target the 3'-UTR of genes' mRNAs to suppress their translation or degrade them. To investigate the molecular mechanism of miR-125b-2-3p, we predicted the target gene of miR125b-2-3p based on the online TargetScan V7.2 software. Slug was selected as a candidate gene for its high binding score and 8mers seed sequences (Supplementary Fig. S3). Dual-luciferase reporter assay was performed and the results showed that obviously reduced luciferase activity was observed in Slug-wild-type, whereas no remarkable change of luciferase activity in Slug-mutation type (Fig. $4 a)$, which meant miR-125b-2-3p could bind on the $3^{\prime}$ UTR of Slug mRNA. Furthermore, overexpression of miR-125b-2-3p decreased the protein level of Slug, while the result was just the opposite after knocking down miR125b-2-3p (Fig. 4b). However, Slug mRNA had no remarkable change no matter whether miR-125b-2-3p was overexpressed or low-expressed (Supplementary Fig. S4), which indicated that miR-125b-2-3p inhibit the translation of Slug mRNA.

\section{Slug was overexpressed in OSCC and silencing Slug inhibited migration and invasion in OSCC cells}

Slug is an epithelial-mesenchymal transition (EMT)related transcription factor that could be involved in the invasion and distant metastasis of various tumor cells including $\mathrm{OSCC}^{20,21}$. To verified the potential biological functions of Slug in OSCC, Slug expression profiles, and corresponding clinical data of 319 OSCC patients and 32 normal controls were downloaded from TCGA database. Bioinformatics analysis revealed that Slug expression was remarkably upregulated $(p<0.001)$ (Fig. 4c) and higher expression of Slug meant poor survival and prognosis $(p=0.02847)$ (Fig. $4 \mathrm{~d})$. To further validate these results, the Slug expression was detected in OSCC tumor tissues and matched ANTs with immunohistochemistry. Compared with the ANTs, Slug expression was significantly higher in OSCC tumor tissues (Fig. 4e). Clinicopathological characteristics analysis showed that high expression of Slug was positively correlated with TNM classification $(p=0.0301)$, lymph node metastasis $(p=$ $0.0306)$, and differentiation in OSCC patients $(p=0.0465)$ (Supplementary Table S4). Furthermore, we investigated the expression of Slug, $\beta$-catenin, and some EMT-related markers (E-cadherin, $\mathrm{N}$-cadherin, and vimentin) after knocking down Slug by specific siRNAs (si-Slug 1, si-Slug 2, si-Slug 3) against Slug transcript in OSCC cells. E-cadherin was significantly upregulated while $\mathrm{N}$-cadherin, vimentin, $\beta$-catenin had no obvious change (Fig. 4f). Finally, transwell assays indicated that the deletion of Slug could remarkably inhibit the ability of migration and invasion in OSCC cells (Fig. 4g).

\section{AC007271.3 promoted migration and invasion via miR- 125b-2-3p/Slug axis in OSCC cells}

The biological function and molecular role of lncRNAs are closely associated with their subcellular localization. We previously identified that AC007271.3 was predominantly located in the cell cytoplasm by using RNAFISH assay, which indicated that AC007271.3 might function as a ceRNA of miR-125b-2-3p and regulate Slug. To further determine the relationship between AC007271.3, miR-125b-2-3p, and Slug, first, Pearson's correlation analysis was performed to uncover the association between AC007271.3 and miR-125b-2-3p expression in 82 OSCC tissues. The results indicated that the expression of miR-125b-2-3p was inversely correlated with AC007271.3 (Fig. 5a). Then, the upregulation of Slug and downregulation of E-cadherin were observed when overexpressing AC007271.3, whereas knocking down AC007271.3 resulted in the opposite results (Fig. 5b). In addition, the upregulated Slug and downregulated Ecadherin caused by AC007271.3 was reversed when cotransfecting with miR-125b-2-3p mimic (Fig. 5c). Simultaneously, migration and invasion experiments revealed 

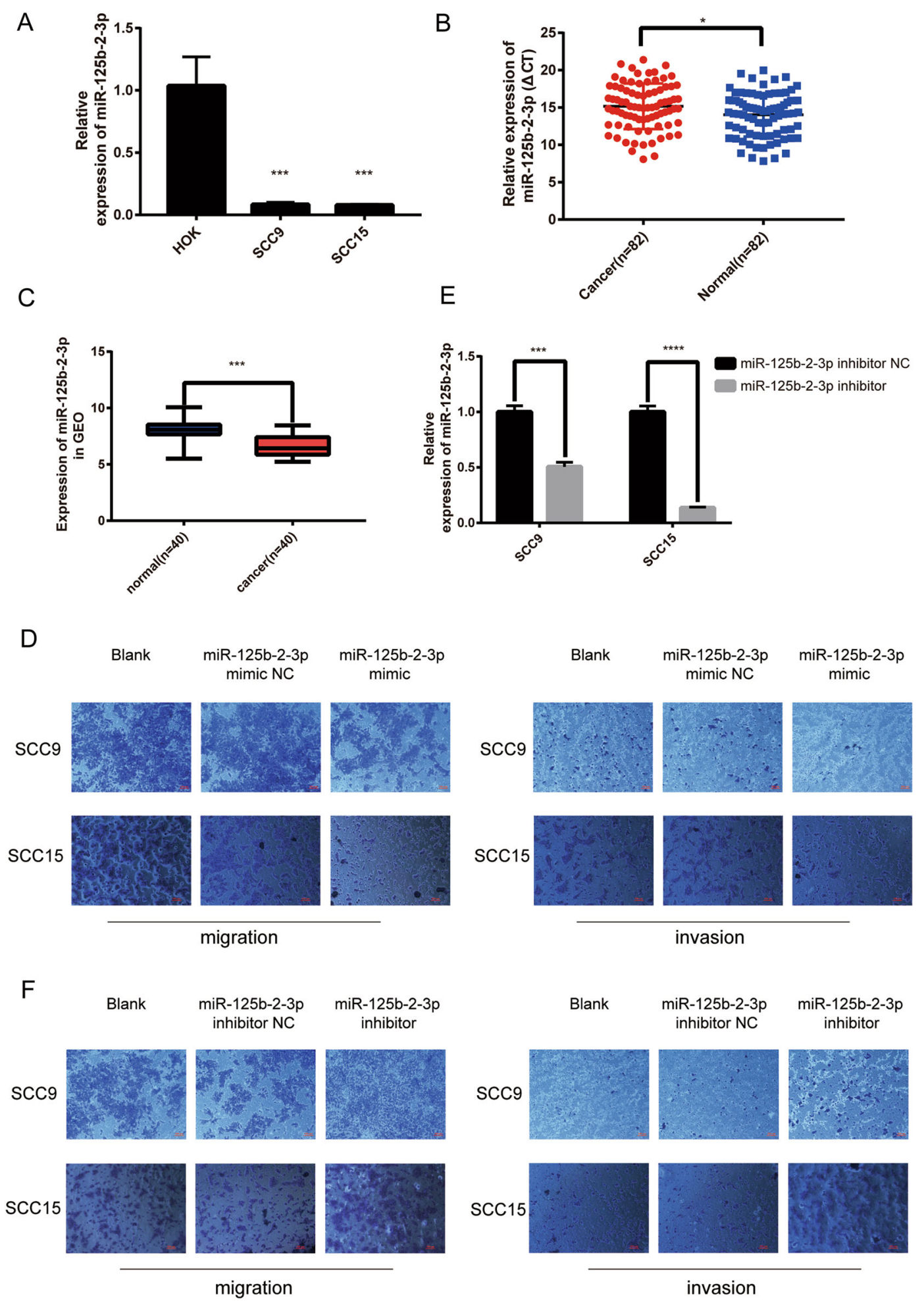

Fig. 3 MiR-125b-2-3p regulated migration and invasion in OSCC cells. a Lower expression of miR-125b-2-3p was detected in two OSCC cells compared to HOK cells. b Differential expression of miR-125b-2-3p in 82 pairs of OSCC clinical samples (Higher $\Delta C T$ means the lower relative expression of miR-125b-2-3p). c The expression data of miR-125b-2-3p in 40 pairs of OSCC in GEO database. d Overexpressing miR-125b-2-3p inhibited the migration (left) and invasion (right) in SCC9 and SCC15. e The inhibition efficiency of miR-125b-2-3p inhibitor in SCC9 and SCC15 cells. f Inhibiting the expression of miR-125b-2-3p promoted the migration (left) and invasion (right) ability in SCC9 and SCC15. ${ }^{*} p<0.05$, ${ }^{* *} p<0.01$, *** $p<$ $0.001,{ }^{* * *} p<0.0001$. 
A

$\begin{array}{llll}\text { Slug-WT } & 5^{\prime} \\ \text { miR-125b-2-3p } & 3^{\prime} \text { CAGGGUUCUCGGACUGAACACU } \\ \text { Slug-MUT } & 5^{\prime} & \text {...GAAGACTGTCGAA... 3' }\end{array}$

B
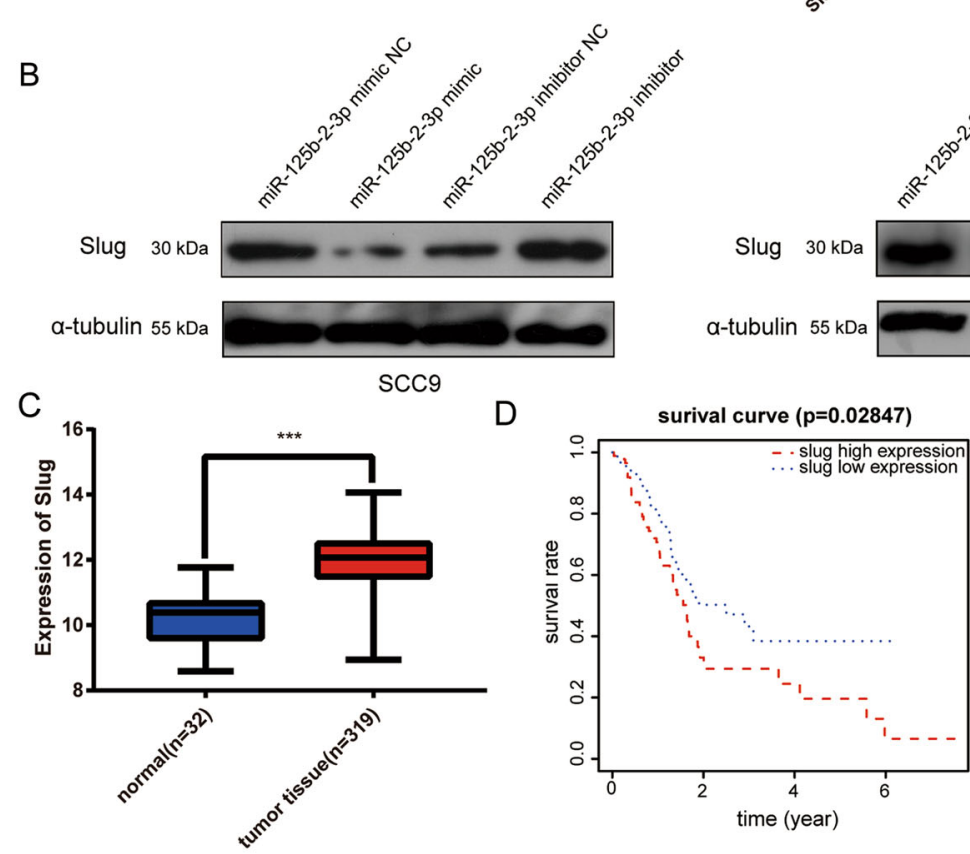

F

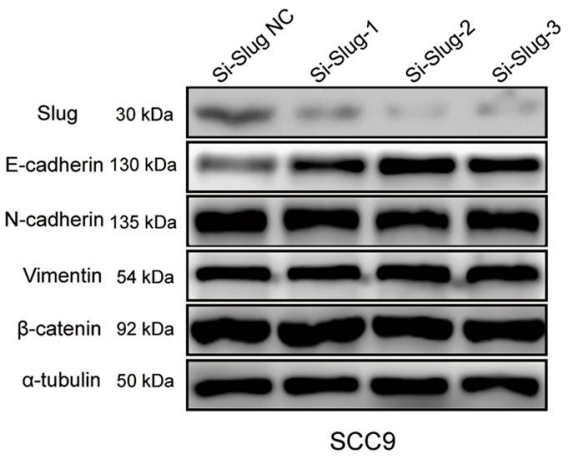

G

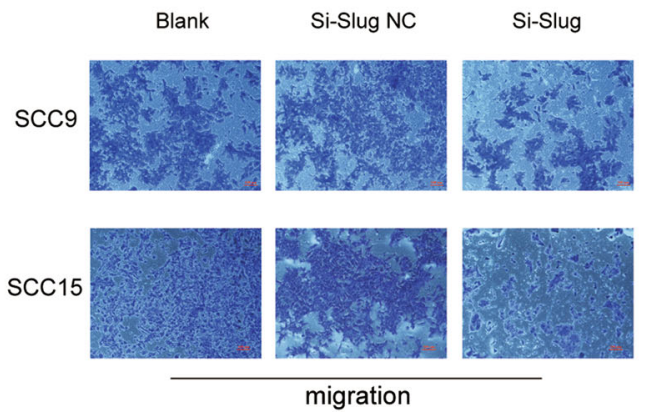

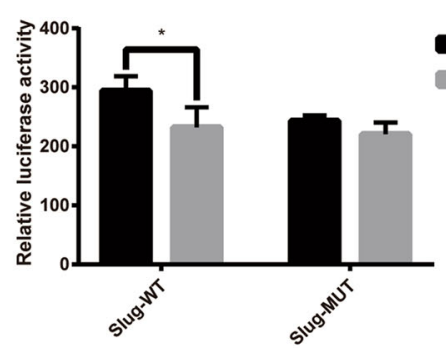

E

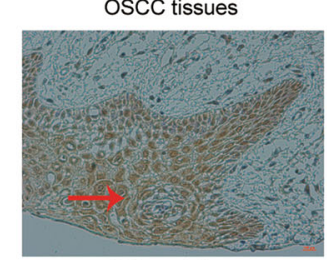

Adjacent tissues

miR-125b-2-3p mimic NC miR-125b-2-3p mimic

Slug $30 \mathrm{kDa}$

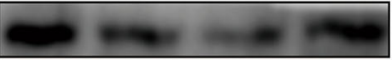

a-tubulin $55 \mathrm{kDa}$
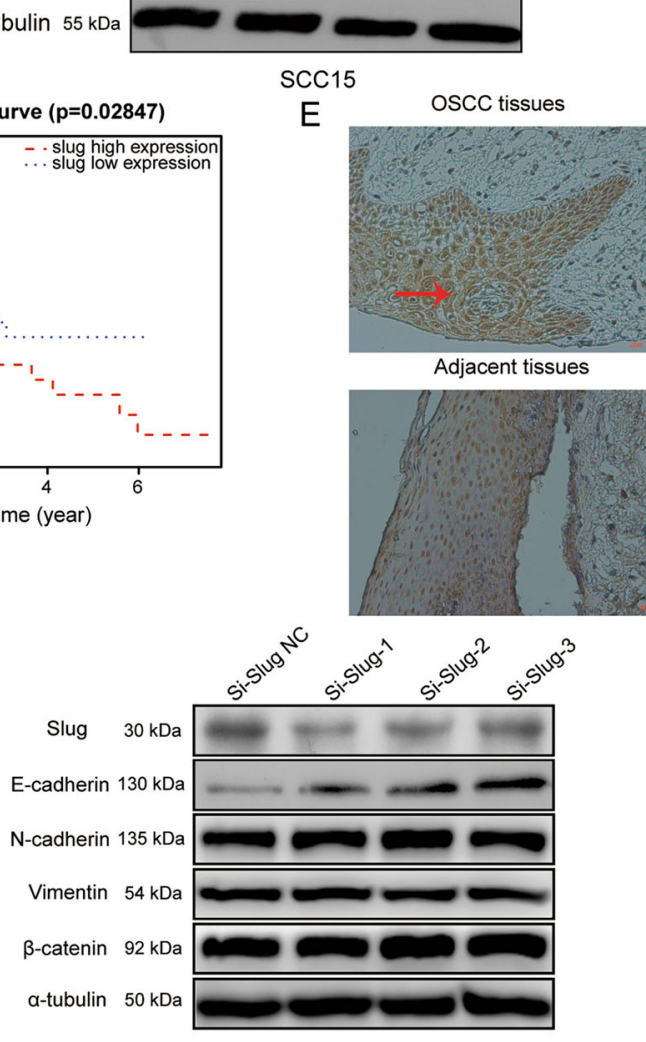

$\operatorname{SCC} 15$

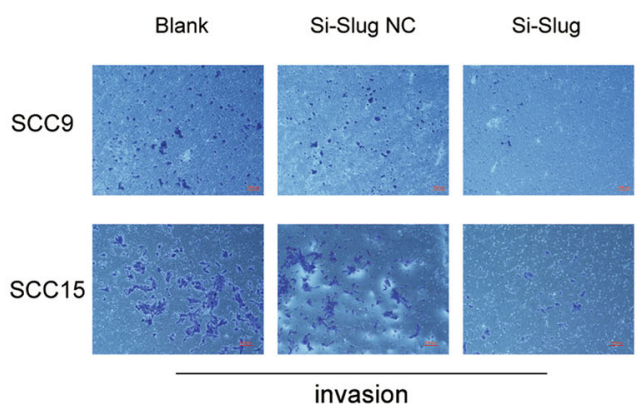

Fig. 4 (See legend on next page.) 
(see figure on previous page)

Fig. 4 Slug was a target gene of miR-125b-2-3p and positively correlated with the malignancy of OSCC. a MiR-125b-2-3p overexpression reduced the relative luciferase activity in Slug-WT. b Overexpressing or inhibiting miR-125b-2-3p changed the expression of Slug protein in SCC9 (left) and SCC15(right). $\mathbf{c}$ The expression of Slug in OSCC $(n=319)$ and normal $(n=32)$ tissues in TCGA database. $\mathbf{d}$ Kaplan-Meier analysis were employed to estimate the relation between Slug expression and overall survival of OSCC patients. e Representative images of Slug expression in the OSCC tumor and adjacent non-malignant tissue analyzed by immunohistochemical. f After transfecting si-Slug in SCC9 and SCC15, the expression of Slug, E-cadherin, N-cadherin, vimentin, and $\beta$-catenin was detected by western blot. $\mathbf{g}$ Si-Slug inhibited the migration(left) and invasion(right) in SCC9 and SCC15. ${ }^{*} p<0.05,{ }^{* *} p<0.01,{ }^{* *} p<0.001$.

that miR-125b-2-3p mimic and si-Slug could reverse the effects resulted from AC007271.3 overexpression (Fig. $5 \mathrm{~d}-\mathrm{e})$.

\section{Upregulation of AC007271.3 promoted OSCC metastasis in vivo}

A pulmonary metastasis model was established to evaluate the effect of AC007271.3 on OSCC metastasis in vivo. We could clearly see more pulmonary metastatic nodules in SCC9-AC007271.3 group than in SCC9-NC group, which suggests that AC007271.3 could significantly promote OSCC metastasis to lung tissues (Fig. 6a-b). QRT-PCR analysis showed that the expression of AC007271.3 in pulmonary metastases was dramatically increased, whereas miR-125b-2-3p's RNA level was significantly decreased in the SCC9-AC007271.3 group in contrast to that in the SCC9-NC group (Fig. 6c). Furthermore, H\&E staining of lung sections indicated that overexpression of AC007271.3 increased the number and size of pulmonary metastases. The results of IHC staining and western blot also revealed a significant upregulation of Slug in SCC9-AC007271.3 group (Fig. 6d-e). These results indicated that AC007271.3 contributed to OSCC metastasis in vivo.

\section{The expression of AC007271.3 was promoted by canonical NF-KB pathway}

In order to explore the upstream regulation mechanism of AC007271.3, dual-luciferase activity assay was performed for the identification of core promoter region of AC007271.3. The unidirectional deletion at $-1000 /-519$ caused a significant reduction in the luciferase activity (Fig. 7a) so we preliminarily regarded $-1000 /-519$ region as the core promoter of AC007271.3. Next, we predicted that NF- $\mathrm{KB}$ may be the potential transcription factor on the core promoter of AC007271.3 on Gene-regulation database. NF- $\mathrm{KB}$ is a dimer formed by different kinds of subunits. The prediction on JASPAR database indicated that NFKB1 may be the most critical subunit (Fig. 7b, Supplementary Table S5). To verify our conjecture, we designed three pairs of primers of the predicted binding sites (AC007271.3-promoter Site 1: -953/-943, AC007271.3-promoter Site 2: -854/-842, AC007271.3promoter Site 3: $-581 /-571)$. ChIP-qPCR analysis confirmed that NFKB1 enriched on the AC007271.3promoter Site 2 (Fig. 7c). NFKB1 is also known as p50, and p50/p65 is the most common type of NF- $\mathrm{kB}$ dimer, which is activated by TNF- $\alpha$ in the classical NF- $\kappa B$ pathway and plays a positive regulatory role during gene transcription. Therefore, we measured the mRNA level of AC007271.3, miR-125b-2-3p, and Slug after TNF- $\alpha$ treatment for the indicated times. The mRNA level of AC007271.3 and Slug were stably activated after $0.5 \mathrm{~h}$ and $2 \mathrm{~h}$, respectively. On the other hand, interestingly, the mRNA level of miR-125b-2-3p strongly increased after $0.5 \mathrm{~h}$ but gradually decreased with the increasing time until $72 \mathrm{~h}$ (Fig. 7d). Western blot indicated that the protein level of Slug was also upregulated after TNF- $\alpha$ treatment (Fig. 7e), and at the same time, nuclear translocation of p65 and nuclear-phosphorylation of p50 and p65 were detected (Fig. 7f). These results suggested that the activation of canonical NF- $\mathrm{kB}$ pathway could positively regulate AC007271.3 and affect the expression of miR-125b-2-3p and Slug.

\section{Discussion}

Accumulating evidence indicated that lncRNAs are closely related to tumorigenesis and development. Our recent studies confirmed that aberrant AC007271.3 level in OSCC patients was significantly associated with clinical stage, especially in early-stage disease and serum AC007271.3 levels could also discriminate between OSCC and normal controls with high sensitivity and specificity, which suggesting that AC007271.3 could be a novel circulating biomarker for the determination of $\mathrm{OSCC}^{11}$. Furthermore, we found that AC007271.3 could promote cell proliferation, invasion, and inhibit cell apoptosis of OSCC via the $\mathrm{Wnt} / \beta$-catenin signaling pathway, which might provide a novel therapeutic approach for $\mathrm{OSCC}^{12}$. In this study, we found that overexpression of AC007271.3 could facilitate the metastasis of OSCC cells in vivo, which further demonstrated the tumorigenesis role of AC007271.3. However, the underlying mechanism of AC007271.3 in OSCC carcinogenesis keep unclear.

LncRNAs' functions are closely associated with its subcellular localization ${ }^{22}$. Plenty of evidence has indicated that IncRNAs can regulate target genes' expression by functioning as ceRNAs of miRNAs ${ }^{15,23}$. We previously 


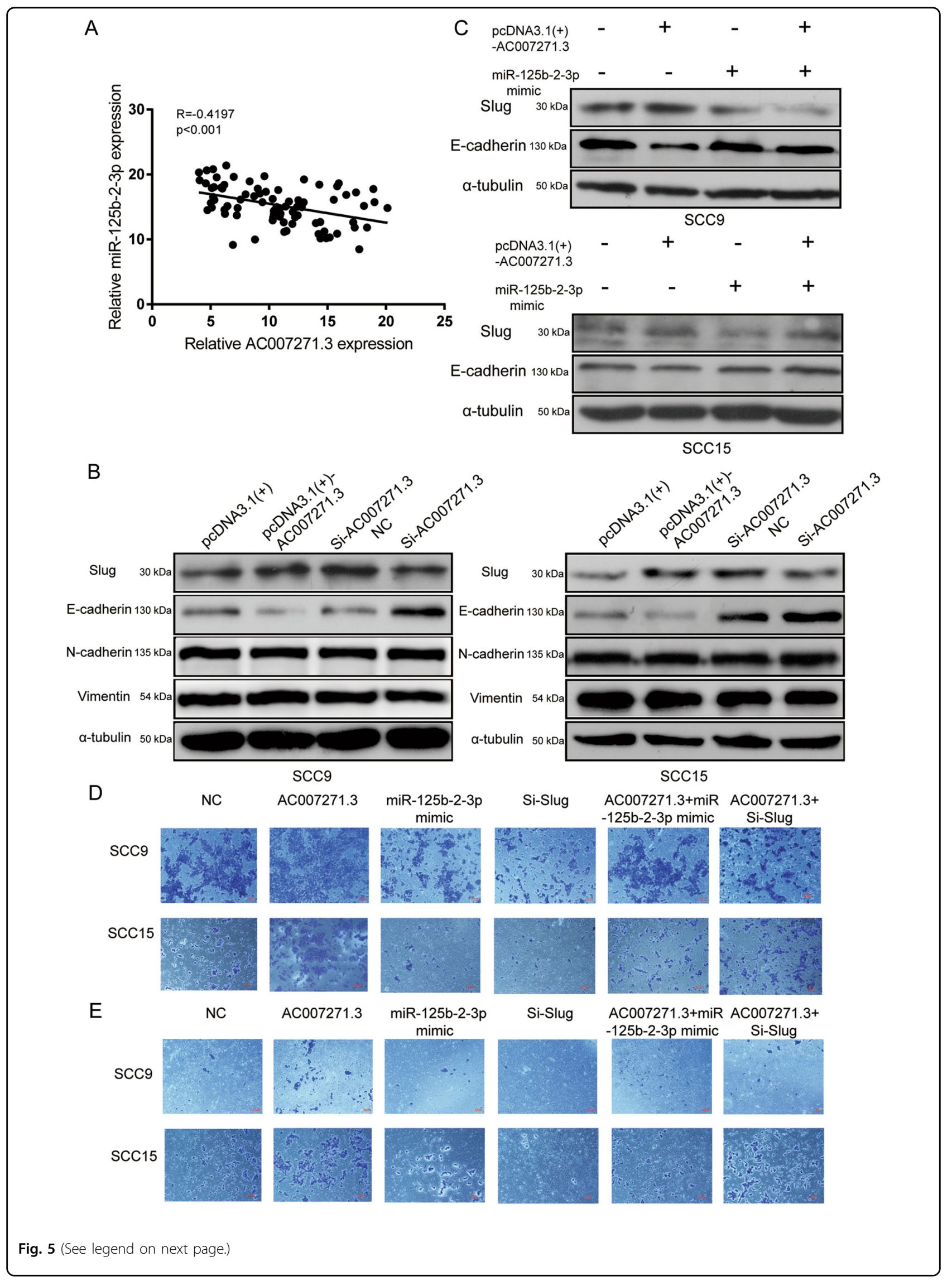


(see figure on previous page)

Fig. 5 AC007271.3 promoted migration and invasion via miR-125b-2-3p/Slug in OSCC cells. a Pearson's correlation analysis of the relationship between AC007271.3 and miR-125b-2-3p ( $n=82)$. $\mathbf{b}$ Western blot analysis was performed to detect the expression of Slug and EMT-related markers after overexpressing (or knocking down) AC007271.3 in SCC9 and SCC15. c MiR-125b-2-3p mimic could reverse the changing expression of Slug and E-cadherin caused by overexpressed AC007271.3. $\mathbf{d}$-e Effects of pcDNA3.1(+)-AC007271.3, miR-125b-2-3p mimic, si-Slug on the migration $\mathbf{d}$ and invasion e in SCC9 and SCC15.

proved that AC007271.3 was mainly located in the cytoplasm and partly in the nucleus. Thus, it indicated that AC007271.3 may mainly function as an endogenous miRNA sponge to regulate the expression of target genes. In the present study, bioinformatics analysis, luciferase reporter, and RNA pulldown assays revealed that miR125b-2-3p was a direct target of AC007271.3. Moreover, we interestingly found an inter-regulatory relationship between the expression of AC007271.3 and miR-125b-2$3 p$. This similar phenomenon was also reported in some researches about ceRNA ${ }^{17,24}$. However, the exact mechanisms were still unknown. In another study, it was reported that lncRNA uc.173 can induce degradation of miR-195 by destabilized its precursor ${ }^{25}$. Therefore, we speculated that whether AC007271.3 could downregulate miR-125b-2-3p through this mechanism and the results confirmed our speculation. To our knowledge, this is the first report to manifest that AC007271.3 could not only played as a ceRNA of miR-125b-2-3p, but also regulated miR-125b-2-3p by destabilized pri-miR-125b-2 in OSCC.

MiR-125b-2-3p is a member of human miR-125 family, which consists of three homologs (miR-125a, miR-125b-1, and miR-125b-2). The miR-125b-2-3p was derived from the 3 'arm of the precursor miRNA(pre-miRNA) of miR$125 b-2^{26}$. It had reported that miR-125b-2-3p was downregulated in hepatocellular carcinoma ${ }^{27}$ and small cell osteosarcoma ${ }^{28}$ but upregulated in tumor stroma of colon cancer ${ }^{29}$. In OSCC, although miR-125b was found to inhibit the progression of OSCC ${ }^{30}$, the function of miR$125 b-2-3 p$ is still uncertain. In this study, we predicted and confirmed that miR-125b-2-3p directly regulated Slug to function as a OSCC tumor suppressor.

Slug, also known as Snail Family Transcriptional Repressor 2 (Snai2), is a member of the Snail family (Snai1, Snai2, Snai3). It is an important transcription factor closely relates to the occurrence and development of various diseases including cancer ${ }^{31}$. Aberrant expression of Slug has been closely related to cancer stem cell formation, cell cycle regulation, and apoptosis as well as invasion and metastasis ${ }^{21}$. As we predicted in the present study, Slug was a target gene of miR-125b-2-3p by bioinformatics analysis and then verified by luciferase reporter assays and western blot. The expression of Slug was remarkably upregulated in OSCC tissues than in normal controls and meant unfavorable prognosis by bioinformatics analysis. We further found that Slug expression was significantly higher in OSCC tissues than in the ANTs by immunohistochemistry and high expression of Slug was positively correlated with TNM classification, lymph node metastasis, and differentiation in OSCC patients. In addition, knockdown of Slug expression remarkably impaired the ability of migration and invasion of OSCC cells. These results discovered that Slug might play an important role in OSCC carcinogenesis. Furthermore, we found that AC007271.3 positively regulated the expression of Slug. In the rescue experiment, overexpression of miR-125b-2-3p reversed the upregulated Slug caused by overexpressed AC007271.3. Simultaneously, migration and invasion experiments unveiled that miR-125b-2-3p mimic and si-Slug can reverse the effects resulted from AC007271.3 overexpression. Taken together, AC007271.3 could regulate Slug expression by regulating miR-125b-2-3p.

Snai family members could directly bind to the promoter of E-cadherin by E-box region and negatively regulate its expression $^{32,33}$. E-cadherin, $\mathrm{N}$-cadherin, and vimentin were the frequent symbol markers for $\mathrm{EMT}^{34}$, which could enhance the invasion and migration of tumors. In this work, we found that the knockdown of Slug induced no significant changes on $\mathrm{N}$-cadherin, vimentin, and $\beta$-catenin except a remarkable decrease of E-cadherin, which indicated that Slug could change the EMT phenotype by inhibiting the expression of E-cadherin and then promoted the migration and invasion in OSCC cells. On the other hand, the E-cadherin/catenin complex, which binds to cytoskeletal components, is an important regulator to form a mature adherent junction ${ }^{35}$. The reduction in E-cadherin was related to the activating of $\beta$-catenin in colorectal cancer $^{36}$. Combined with our results, we speculated that Slug may increase the dissociated $\beta$-catenin, which could be activated and translocated into the nucleus, by reducing the expression of Ecadherin. Moreover, recent researches implied that the Wnt/ $\beta$-catenin pathway participated in the regulation of $\operatorname{Slug}^{37,38}$, which may form a feed-back regulation between Slug/E-cadherin/ $\beta$-catenin. However, the relation between Slug, E-cadherin, and $\beta$-catenin in OSCC needs to be further explored.

$\mathrm{NF}-\mathrm{\kappa B}$ is a regulator of expression of the $\mathrm{\kappa B}$ light chain firstly recognized in $B$ cells. It is a homo- or heterodimer formed by any two of the five subunits of RelA (p65), RelB, c-Rel, NFKB1 (p50), NFKB2 (p52) ${ }^{39}$. Plenty of evidence 


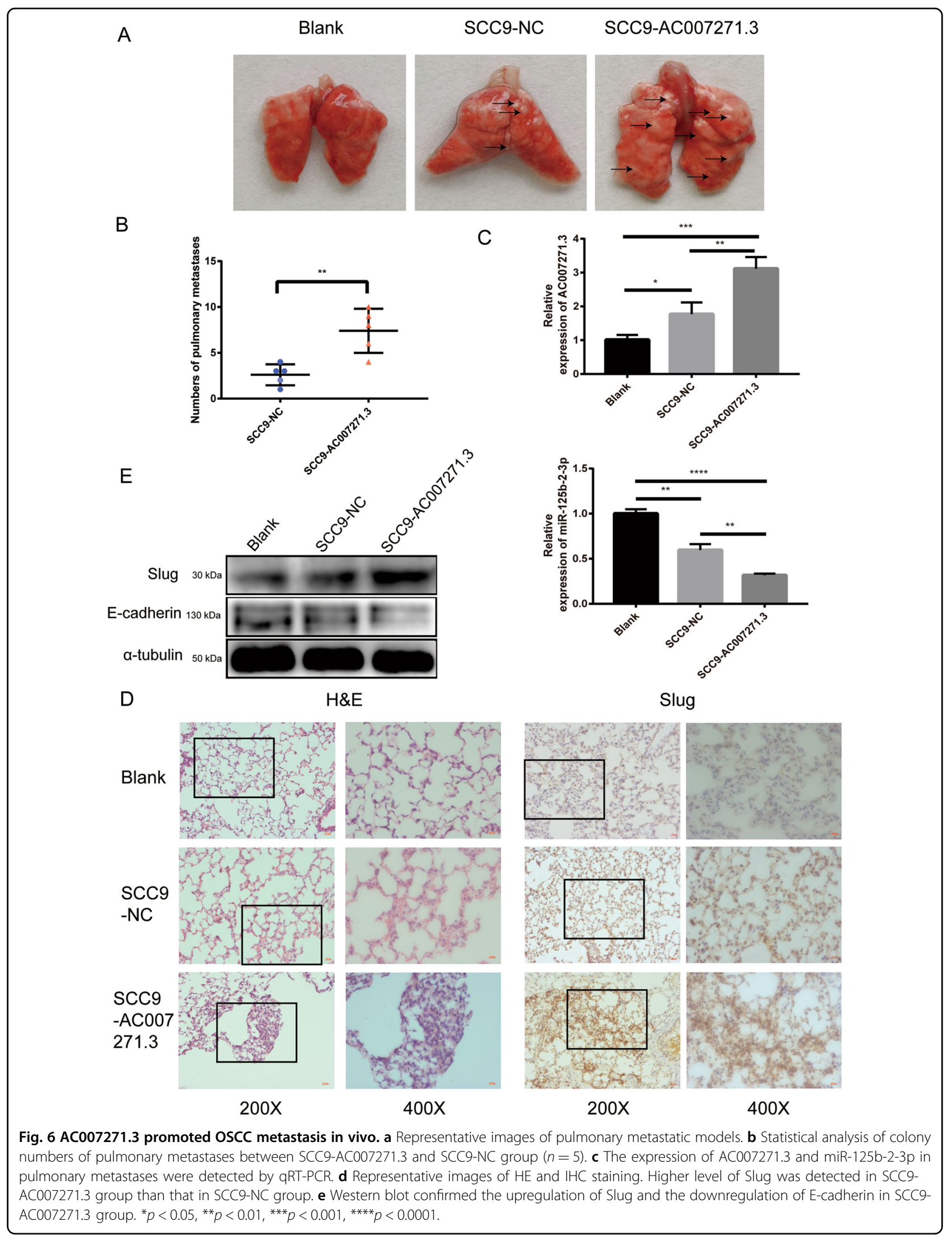



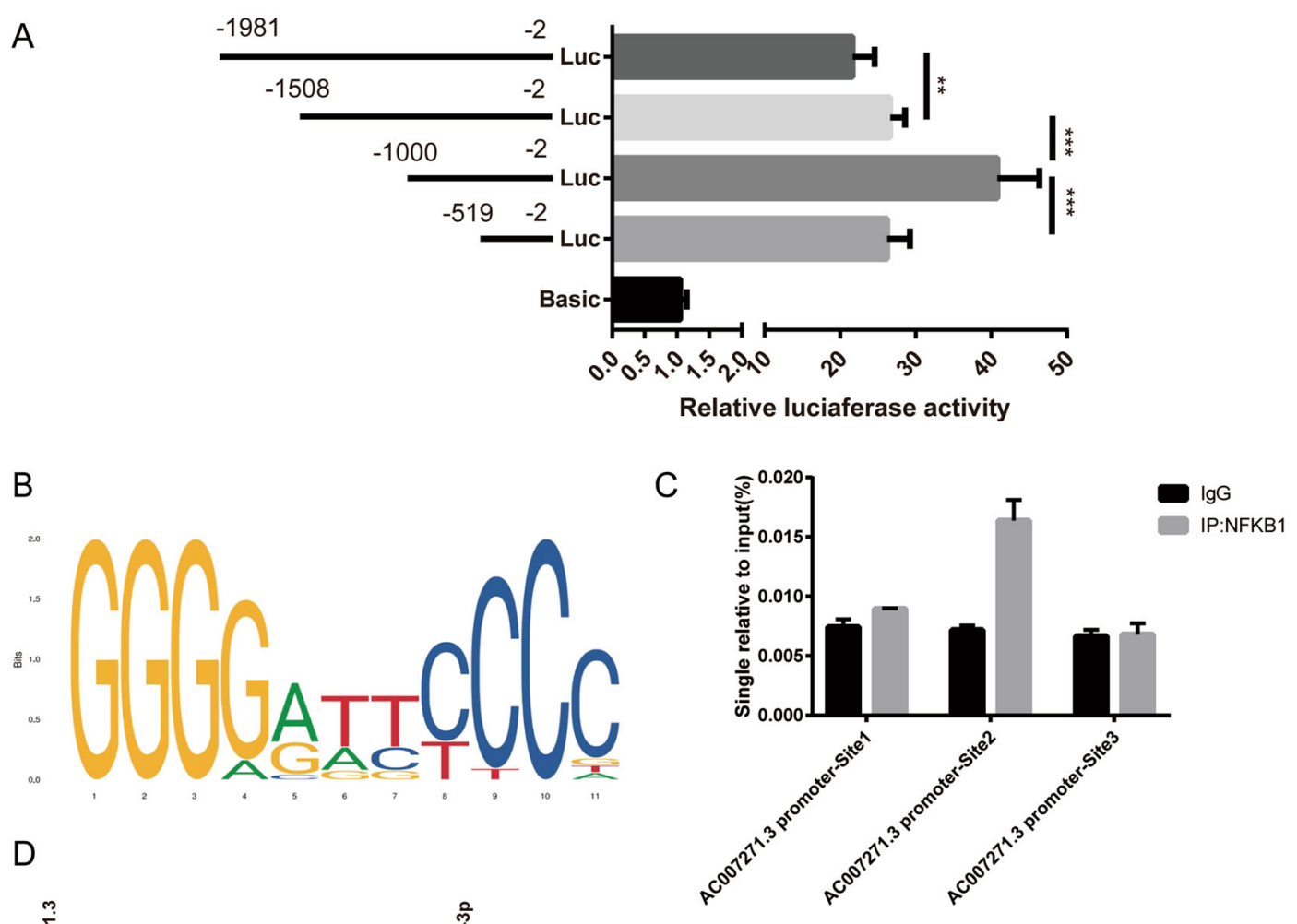

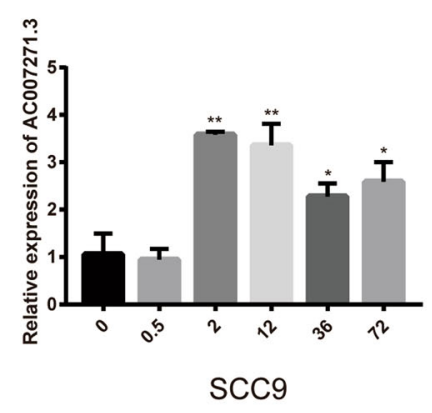

E

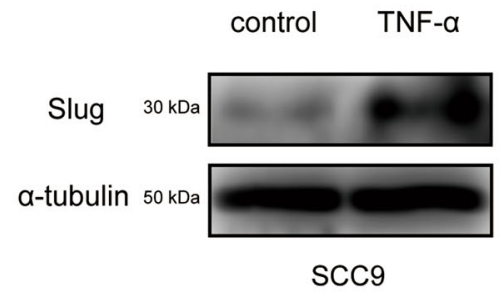

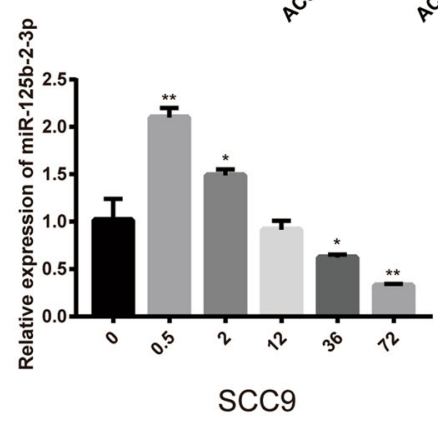

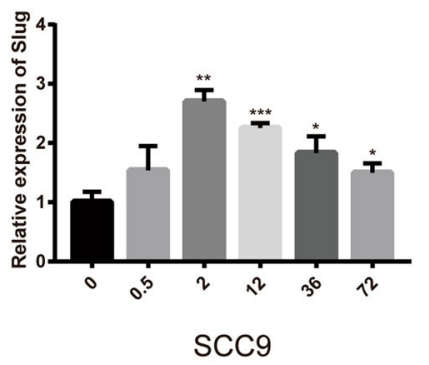

F

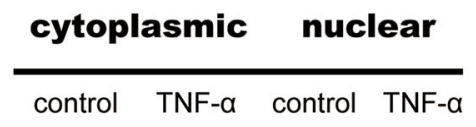

p50 $50 \mathrm{kDa}$

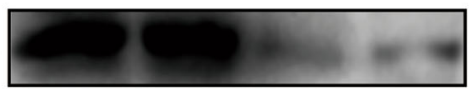

p-p50(Ser 337) $50 \mathrm{kDa}$

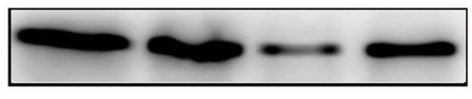

p65 $65 \mathrm{kDa}$

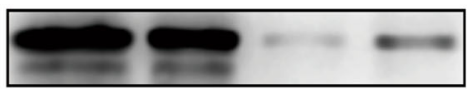

p-p65(Ser 536) $65 \mathrm{kDa}$

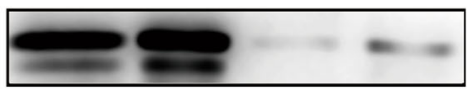

a-tubulin $50 \mathrm{kDa}$

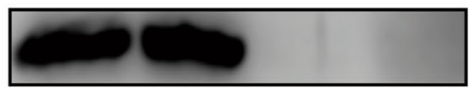

Histon $\mathrm{H} 3 \quad 17 \mathrm{kDa}$

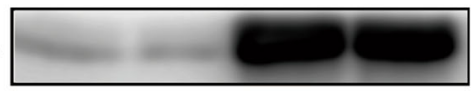

$\operatorname{scc} 9$

Fig. 7 (See legend on next page.) 
(see figure on previous page)

Fig. 7 NFKB1 was enriched on the core promoter region of AC007271.3 and the canonical NF-KB pathway upregulated the expression of AC007271.3. a After transfecting fragment-by-fragment deletion PGL3 vectors of AC007271.3 promoters into $293 \mathrm{~T}$ cells for 48 hours, the luciferase activity was measured and analyzed statistically. $\mathbf{b}$ The binding sequence logo of NFKB1 on JASPAR database. $\mathbf{c}$ Enrichment of NFKB1 was detected at the Site 2 (-854/-842) of AC007271.3 core promoter in SCC9 by ChIP-qPCR analysis. $\mathbf{d}$ After treating with TNF-a $(10 \mathrm{ng} / \mathrm{ml})$ for the indicated times in SCC9, the mRNA level of AC007271.3, miR-125b-2-3p, and Slug was analyzed by qRT-PCR. GAPDH was used as a control. e The western blot analysis indicated that the protein level of Slug also increased by TNF-a treatment (10 ng/ml, 72 hours). a-tubulin was employed as a control. $\mathbf{f}$ The canonical NF-kB pathway-related proteins in the cytoplasmic and nuclear of SCC9 which treated with or without TNF-a $(10 \mathrm{ng} / \mathrm{ml}, 72 \mathrm{~h})$ were assessed by western blot. a-tubulin and Histone H3 were employed as the positive controls for cytoplasmic and nuclear proteins, respectively. ${ }^{*} p<0.05,{ }^{* *} p<0.01,{ }^{* * *} p<0.001$.

indicated that NF- $\mathrm{BB}$ pathway played an important role in inflammation and cancer ${ }^{40,41}$. In the canonical NF-kB pathway, tumor necrosis factor (TNF)- $\alpha$ stimulation induces IKK $\beta$ phosphorylation and then phosphorylates IкB $\alpha$ to promote its polyubiquitination and degradation, which lead to the release of $\mathrm{p} 65 / \mathrm{p} 50$ heterodimer. $\mathrm{P} 65 / \mathrm{p} 50$ dimers may be phosphorylated ${ }^{42}$ and then translocate into the nucleus and bind to the specific DNA sequences to promote the transcription of target genes ${ }^{43}$. The NF- $\mathrm{BB}$ pathway has been fully studied in the past few decades. It was widely acknowledged that $\mathrm{p} 50 / \mathrm{p} 65$ dimer plays a direct role in the canonical NF- $\mathrm{kB}$ pathway to positively regulate the transcription of target genes ${ }^{39-41,43}$. Phosphorylation sites of p50 and p65 also regulate the function of this dimer ${ }^{42,44}$. Previous studies showed that p50 (Ser337) site phosphorylation could enhance the DNA binding ability of $\mathrm{p} 50^{45}$, whereas p65 (Ser536) phosphorylation could enhance the transactivation potential of $\mathrm{p} 65^{46}$. In the research, after treatment of TNF- $\alpha$ in SCC9, the overexpressed AC007271.3 were detected, and the p50 (Ser337) and p65 (Ser536) were significantly upregulated in the nucleus. These results suggested that the activation of the canonical NF- $\kappa B$ pathway could positively regulate the expression of AC007271.3. In addition, a recent study showed that NF- $\mathrm{kB}$ pathway promoted metastasis in head and neck squamous cell carcinoma cells by stabilizing Slug ${ }^{47}$. However, the underlying mechanism is not clear. Our results identified that the classical NF- $\mathrm{kB}$ pathway probably regulated the migration and invasion of OSCC through the AC007271.3/ miR-125b-2-3p/Slug axis. To our knowledge, it is the first time to propose this potential mechanism in OSCC.

\section{Conclusion}

In conclusion, our research confirmed that the classical NF- $\kappa B$ pathway-regulated AC007271.3 interacted with miR-125b-2-3p to regulate Slug gene and promote the migration and invasion in OSCC cells, which suggested that AC007271.3 may be a diagnostic molecule and therapeutic target for OSCC.

\section{Acknowledgements}

This study was supported by the National Natural Science Foundation of China (81472536); the Science and Technology Planning Project of Guangdong Province (no. 2017A020215181); the Southern Medical University Scientific Research Fund (CX2018N016); Project of Educational Commission of
Guangdong Province of China (2018KTSCX026); and the Presidential Foundation of the Nanfang Hospital (2014027, 2019Z030).

\section{Conflict of interest}

The authors declare that they have no conflict of interest.

\section{Publisher's note}

Springer Nature remains neutral with regard to jurisdictional claims in published maps and institutional affiliations.

Supplementary Information accompanies this paper at (https://doi.org/ 10.1038/s41419-020-03257-4).

Received: 21 August 2020 Revised: 16 November 2020 Accepted: 17 November 2020

Published online: 12 December 2020

\section{References}

1. Bray, F. et al. A. Global cancer statistics 2018: GLOBOCAN estimates of incidence and mortality worldwide for 36 cancers in 185 countries. CA Cancer J. Clin. 68, 394-424 (2018).

2. Chi, A. C., Day, T. A. \& Neville, B. W. Oral cavity and oropharyngeal squamous cell carcinoma-an update. CA Cancer J. Clin. 65, 401-421 (2015).

3. Khan, T. et al. Autophagy modulators for the treatment of oral and esophageal squamous cell carcinomas. Med. Res. Rev. 40, 1002-1060 (2019).

4. Chen, I. C. et al. Role of SIRT1 in regulation of epithelial-to-mesenchymal transition in oral squamous cell carcinoma metastasis. Mol. Cancer 13, 254 (2014).

5. Quinn, J. J. \& Chang, H. Y. Unique features of long non-coding RNA biogenesis and function. Nat. Rev. Genet. 17, 47-62 (2016).

6. Schmitt, A. M. \& Chang, H. Y. Long noncoding RNAs in cancer pathways. Cancer Cell 29, 452-463 (2016).

7. Kopp, F. \& Mendell, J. T. Functional classification and experimental dissection of long noncoding RNAs. Cell 172, 393-407 (2018).

8. Zhang, L. et al. Long non-coding RNAs in oral squamous cell carcinoma: biologic function, mechanisms and clinical implications. Mol. Cancer 18, 102 (2019).

9. Ding, L. et al. A novel stromal IncRNA signature reprograms fibroblasts to promote the growth of oral squamous cell carcinoma via LncRNA-CAF/ interleukin-33. Carcinogenesis 39, 397-406 (2018).

10. Yang, Y., Chen, D., Liu, H. \& Yang, K. Increased expression of IncRNA CASC9 promotes tumor progression by suppressing autophagy-mediated cell apoptosis via the AKT/mTOR pathway in oral squamous cell carcinoma. Cell Death Dis. 10, 41 (2019).

11. Shao, T. et al. SCCA, TSGF, and the long non-coding RNA AC007271.3 are effective biomarkers for diagnosing oral squamous cell carcinoma. Cell Physiol. Biochem. 47, 26-38 (2018).

12. Shao, T. R. et al. LncRNA AC007271.3 promotes cell proliferation, invasion, migration and inhibits cell apoptosis of OSCC via the Wnt/beta-catenin signaling pathway. Life Sci. 239, 117087 (2019).

13. Bartel, D. P. MicroRNAs: genomics, biogenesis, mechanism, and function. Cell 116, 281-297 (2004).

14. Rupaimoole, R. \& Slack, F. J. MicroRNA therapeutics: towards a new era for the management of cancer and other diseases. Nat. Rev. Drug Discov. 16, 203-221 (2017). 
15. Thomson, D. W. \& Dinger, M. E. Endogenous microRNA sponges: evidence and controversy. Nat. Rev. Genet 17, 272-283 (2016).

16. Zhang, G. et al. LncRNA MT1JP functions as a ceRNA in regulating FBXW7 through competitively binding to miR-92a-3p in gastric cancer. Mol. Cancer 17, 87 (2018).

17. Li, Y. et al. Long non-coding RNA SNHG5 promotes human hepatocellular carcinoma progression by regulating miR-26a-5p/GSK3beta signal pathway. Cell Death Dis. 9, 888 (2018).

18. Fang, Z. et al. LncRNA UCA1 promotes proliferation and cisplatin resistance of oral squamous cell carcinoma by sunppressing miR-184 expression. Cancer Med. 6, 2897-2908 (2017).

19. Hong, $\mathrm{Y}$. et al. Long non-coding RNA H1 promotes cell proliferation and invasion by acting as a ceRNA of miR138 and releasing EZH2 in oral squamous cell carcinoma. Int J. Oncol. 52, 901-912 (2018).

20. Joseph, M. J. et al. Slug is a downstream mediator of transforming growth factor-beta1-induced matrix metalloproteinase-9 expression and invasion of oral cancer cells. J. Cell Biochem. 108, 726-736 (2009).

21. Zhou, W., Gross, K. M. \& Kuperwasser, C. Molecular regulation of Snai2 in development and disease. J. Cell Sci. 132, jcs235127 (2019).

22. Yao, R. W., Wang, Y. \& Chen, L. L. Cellular functions of long noncoding RNAs. Nat. Cell Biol. 21, 542-551 (2019).

23. Huang, G. Z., Wu, Q. Q., Zheng, Z. N., Shao, T. R. \& LV, X. Z. Identification of candidate biomarkers and analysis of prognostic values in oral squamous cell carcinoma. Front Oncol. 9, 1054 (2019).

24. Wang, M. et al. Long noncoding RNA LINC00336 inhibits ferroptosis in lung cancer by functioning as a competing endogenous RNA. Cell Death Differ. 26, 2329-2343 (2019).

25. Xiao, L. et al. Long noncoding RNA uc.173 promotes renewal of the intestinal mucosa by inducing degradation of MicroRNA 195. Gastroenterology 154, 599-611 (2018).

26. Sun, Y. M., Lin, K. Y. \& Chen, Y. Q. Diverse functions of miR-125 family in different cell contexts. J. Hematol. Oncol. 6, 6 (2013).

27. Shi, J. et al. Coordinative control of G2/M phase of the cell cycle by noncoding RNAs in hepatocellular carcinoma. PeerJ 6, e5787 (2018).

28. Xie, L. et al. Identification of the miRNA-mRNA regulatory network of small cell osteosarcoma based on RNA-seq. Oncotarget 8, 42525-42536 (2017).

29. Zhou, X. G. et al. Identifying miRNA and gene modules of colon cancer associated with pathological stage by weighted gene co-expression network analysis. OncoTargets Ther. 11, 2815-2830 (2018).

30. Chen, Y. F. et al. miR-125b suppresses oral oncogenicity by targeting the antioxidative gene PRXL2A. Redox Biol. 22, 101140 (2019).
31. Cobaleda, C., Perez-Caro, M., Vicente-Duenas, C. \& Sanchez-Garcia, I. Function of the zinc-finger transcription factor SNAI2 in cancer and development. Annu. Rev. Genet. 41, 41-61 (2007).

32. Hajra, K. M., Chen, D. Y. \& Fearon, E. R. The SLUG zinc-finger protein represses E-cadherin in breast cancer. Cancer Res. 62, 1613-1618 (2002).

33. Yang, M. H. et al. Comprehensive analysis of the independent effect of twist and snail in promoting metastasis of hepatocellular carcinoma. Hepatology $\mathbf{5 0}$, 1464-1474 (2009).

34. Nieto, M. A., Huang, R. Y., Jackson, R. A. \& Thiery, J. P. Emt: 2016. Cell 166, 21-45 (2016).

35. Chen, H. N. et al. PDLIM1 stabilizes the E-cadherin/beta-catenin complex to prevent epithelial-mesenchymal transition and metastatic potential of colorectal cancer cells. Cancer Res 76, 1122-1134 (2016).

36. Huels, D. J. et al. E-cadherin can limit the transforming properties of activating beta-catenin mutations. EMBO J. 34, 2321-2333 (2015).

37. Heuberger, J. \& Birchmeier, W. Interplay of cadherin-mediated cell adhesion and canonical Wnt signaling. Cold Spring Harb. Perspect. Biol. 2, a002915 (2010).

38. Wu, Z. Q. et al. Canonical Wnt signaling regulates Slug activity and links epithelial-mesenchymal transition with epigenetic breast cancer 1, early onset (BRCA1) repression. Proc. Natl. Acad. Sci. USA 109, 16654-16659 (2012).

39. Hayden, M. S. \& Ghosh, S. Shared principles in NF-kappaB signaling. Cell 132 344-362 (2008)

40. DiDonato, J. A., Mercurio, F. \& Karin, M. NF-kappaB and the link between inflammation and cancer. Immunol. Rev. 246, 379-400 (2012).

41. Hoesel, B. \& Schmid, J. A. The complexity of NF-kappaB signaling in inflammation and cancer. Mol. Cancer 12, 86 (2013).

42. Viatour, P., Merville, M. P., Bours, V. \& Chariot, A. Phosphorylation of NF-kappaB and IkappaB proteins: implications in cancer and inflammation. Trends Biochem. Sci. 30, 43-52 (2005).

43. Bakkar, N. \& Guttridge, D. C. NF-kappaB signaling: a tale of two pathways in skeletal myogenesis. Physiol. Rev. 90, 495-511 (2010).

44. Lu, X. \& Yarbrough, W. G. Negative regulation of RelA phosphorylation: emerging players and their roles in cancer. Cytokine Growth Factor Rev. 26 7-13 (2015).

45. Hou, S., Guan, H. \& Ricciardi, R. P. Phosphorylation of serine 337 of NF-kappaB p50 is critical for DNA binding. J. Biol. Chem. 278, 45994-45998 (2003).

46. Sakurai, H., Chiba, H., Miyoshi, H., Sugita, T. \& Toriumi, W. IkappaB kinases phosphorylate NF-kappaB p65 subunit on serine 536 in the transactivation domain. J. Biol. Chem. 274, 30353-30356 (1999).

47. Liu, S. et al. Stabilization of slug by NF-kappaB is essential for TNF-alpha -induced migration and epithelial-mesenchymal transition in head and neck squamous cell carcinoma cells. Cell Physiol. Biochem. 47, 567-578 (2018). 